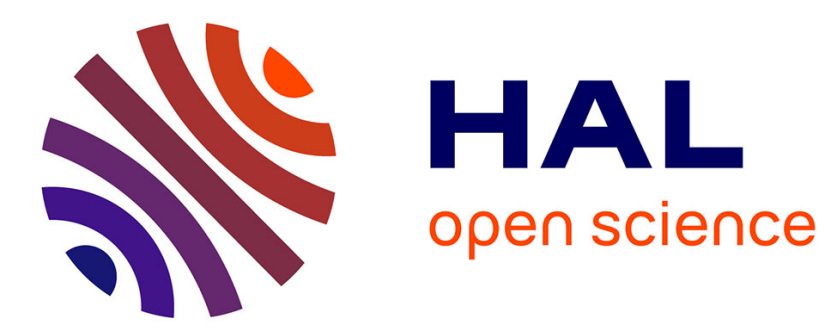

\title{
Meta-parameters Exploration for Unsupervised Event-based Motion Analysis
}

Veis Oudjail, Jean Martinet

\section{To cite this version:}

Veis Oudjail, Jean Martinet. Meta-parameters Exploration for Unsupervised Event-based Motion Analysis. 15th International Conference on Computer Vision Theory and Applications, Feb 2020, Valletta, France. pp.853-860, 10.5220/0009324908530860 . hal-02529895

\section{HAL Id: hal-02529895 https://hal.science/hal-02529895}

Submitted on 2 Apr 2020

HAL is a multi-disciplinary open access archive for the deposit and dissemination of scientific research documents, whether they are published or not. The documents may come from teaching and research institutions in France or abroad, or from public or private research centers.
L'archive ouverte pluridisciplinaire HAL, est destinée au dépôt et à la diffusion de documents scientifiques de niveau recherche, publiés ou non, émanant des établissements d'enseignement et de recherche français ou étrangers, des laboratoires publics ou privés. 


\title{
Meta-parameters exploration for unsupervised event-based motion analysis
}

\author{
Veïs Oudjail ${ }^{1}$, Jean Martinet ${ }^{2}{ }^{\text {a }}$ \\ ${ }^{1}$ Univ. Lille, CNRS, Centrale Lille, UMR 9189 - CRIStAL, F-59000, Lille, France \\ ${ }^{2}$ Université Côte d'Azur, CNRS, I3S, France \\ veis.oudjail@univ-lille.fr, jean.martinet@univ-cotedazur.fr
}

Keywords: $\quad$ Motion Analysis, Spiking Neural Networks, Event-based sensor, parameter exploration.

\begin{abstract}
Being able to estimate motion features is an essential step in dynamic scene analysis. Optical flow typically quantifies the apparent motion of objects. Motion features can benefit from bio-inspired models of mammalian retina, where ganglion cells show preferences to global patterns of direction, especially in the four cardinal translatory directions. We study the meta-parameters of a bio-inspired motion estimation model using event cameras, that are bio-inspired vision sensors that naturally capture the dynamics of a scene. The motion estimation model is made of an elementary Spiking Neural Network, that learns the motion dynamics in a nonsupervised way through the Spike-Timing-Dependent Plasticity. After short simulation times, the model can successfully estimate directions without supervision. Some of the advantages of such networks are the nonsupervised and continuous learning capabilities, and also their implementability on very low-power hardware. The model is tuned using a synthetic dataset generated for parameter estimation, made of various patterns moving in several directions. The parameter exploration shows that attention should be given to model tuning, and yet the model is generally stable over meta-parameter changes.
\end{abstract}

\section{INTRODUCTION}

Motion features are useful in a wide range of computer vision tasks, and traditionally requires the extraction and processing of keyframes, to define motion descriptors such as optical flow. Determining the optical flow consists in estimating the elementary displacements of interest points in a video stream.

High precision approaches are based on deep learning (Ilg et al., 2017). However, most solutions use a large amount of annotated data as part of their supervised learning. Besides, training deep networks use significant computing resources that cause a significant energy cost. Spiking Neural Networks (SNN), however, allow unsupervised learning: the Spike-Timing-Dependent Plasticity (STDP) learning rule used in SNN training is not supervised. Unlike the stochastic gradient descent used in conventional networks, which is a global rule, STDP is local. This locality makes it possible to design lowpower massively parallel hardware circuits. However, the challenge is to be able to use this model in vision tasks with performances that rival state-of-the-art methods. One of the difficulties is the configuration

a (D) https://orcid.org/0000-0001-8821-5556 of the model; indeed, the adjustment is very sensitive and has a direct impact on system performance. A fine-tuning phase must be carried out to find the right setting, which makes it difficult to use SNN.

In this paper, we investigate temporal data analysis with SNN for video. We consider the event cameras such as Dynamic Vision Sensors (DVS) (Lichtsteiner et al., 2008). These sensors output an $\mathrm{Ad}$ dress Event Representation (AER), each pixel individually encodes positive and negative intensity variations - every change triggers an event that is transmitted asynchronously. There are two types of events: $\mathrm{ON}$-events for positive variations and OFF-events for negative variations. Such a representation is well adapted to SNN, because the events resemble spikes, and therefore they can be used to feed the network in a straightforward manner. Our objective is to study elementary network structures able to classify a motion stimulus inside a small window. We successfully trained simple one-layer fully-connected feedforward spiking neural networks to recognise the motion orientation of binary patterns in a $5 \times 5$ window, in an unsupervised manner.

We considered several patterns, with a varying number of input spiking pixels. We explored the 
meta-parameter space in order to exhibit successful settings. Through this exploration, we identified key meta-parameters that have the most impact. We show that the neuron activation threshold is the most influencing meta-parameter for the performance. We also show the need to adjust the threshold value when the number of active input pixels changes.

The remainder of the paper is organised as follows: Section 2 discusses the use of SNN in vision, namely for image and video classification, Section 3 describes the core model, by giving details regarding the dataset, the network structure, and the experimental protocol, Section 4 shows and discusses the metaparameter exploration, and Section 5 concludes the paper and discusses future work.

\section{RELATED WORK}

\subsection{Spiking Neural Networks}

Spiking Neural Networks represent a special class of artificial neural networks (Maass, 1997), where neurons communicate by sequences of spikes (Ponulak and Kasinski, 2011). SNN have long been used in the neuroscience community as a reliable model to precisely simulate biology and understand brain mechanisms (Paugam-Moisy and Bohte, 2012).

Contrary to widely-used deep convolutional neural networks, spiking neurons do not fire at each propagation cycle, but rather fire only when their activation level (or membrane potential, an intrinsic quality of the neuron related to its membrane electrical charge) reaches a specific threshold value. SNN do not rely on stochastic gradient descent and backpropagation. Instead, neurons are connected through synapses, that implement a learning mechanism inspired from biology. The STDP is a rule that updates synaptic weights according to the spike timings, and increases the weight when a presynaptic spike occurs just before a postsynaptic spike (within a few milliseconds). Therefore, the learning process is intrinsically not supervised, and SNN can be successfully used to detect patterns in data in an unsupervised manner (Oudjail and Martinet, 2019; Bichler et al., 2012; Hopkins et al., 2018).

Several studies have attempted to reproduce and apply to SNN several mechanisms that contribute to the success of deep networks, such as the stochastic gradient descent (Lee et al., 2016) or deep convolutional architectures (Cao et al., 2015; Tavanaei and Maida, 2017). However, such approaches do not benefit from the unsupervised advantage of STDP.

\subsection{Event video classification}

In addition, SNN are increasingly used in data processing because of their implementability on low-energy hardware such as neuromorphic circuits (Merolla et al., 2014; Sourikopoulos et al., 2017; Kreiser et al., 2017). SNN have been used in vision-related tasks (Masquelier and Thorpe, 2007), and some researchers have addressed standard vision datasets with SNN and DVS by converting them into an event representation, such as Poker-DVS, MNISTDVS (Serrano-Gotarredona and Linares-Barranco, 2015), N-MNIST (Orchard et al., 2015), and CIFARDVS (Li et al., 2017).

More specifically in motion analysis, several attempts to apply SNN for video classification or for more specific video-related tasks exist. Bichler et al. (Bichler et al., 2012) have used a feed-forward SNN capable of recognising the movement of a ball among 8 discretised directions from event data. They also show in another experiment that SNN can be used to count cars passing on a highway lane. The data is captured with a DVS.

Orchard et al. (Orchard et al., 2013) developed a system to extract the optical flow from an event video sequence. A simple test setup was constructed consisting of a black pipe spinning in front of a white background. This setup is used for testing different motion speeds and directions. In their approach, they use $5 \times 5$ receptive fields, with neurons that are sensitive to certain motion directions and speeds ( 8 directions and 8 speeds), with the assumption that the speed is fixed and constant. In the model, they integrate, synaptic delays in order to be sensitive to spatio-temporal patterns. All parameters are fixed at the beginning, so there is no learning in their model.

Zhao et al. (Zhao et al., 2015) combine an HMAX and SNN feed-forward architecture to recognise the following 3 human actions from event videos: bending, walking and standing/sitting, where the types of movement can be simplified by diagonal, horizontal and vertical motions.

Amir et al. (Amir et al., 2017) have designed a demo for recognising more than 10 hand gestures in real time with a low-energy consumption, by exploiting a neuromorphic processor with a capacity of one million neurons, running an SNN coupled to a pretrained CNN. Such a system reached a $96.5 \%$ success rate.

\subsection{Relation to our work}

All previously mentioned work use both $\mathrm{ON}$ - and OFF-event types. With this rich information, if we 
assume a constant intensity for objects and backgrounds, the object motion can be inferred in short temporal windows. Let us take the example of a white ball moving towards the right before a darker background. The right edge of the ball will generate a set of ON-events and the opposite edge (left) will simultaneously generate a set of OFF events. In this setting, the motion recognition problem boils down to a static pattern recognition problem, because the very motion is encoded in a static way. Moreover, if the object is either large or close to the sensor, then ON-events and OFF-events will be much separated in space, or even separated in time (i.e. they will not occur simultaneously in the sensor) if the object is larger than the sensor's field of view, making it almost impossible for such static approaches to infer a motion. In this paper, we study focus on learning dynamic object motions. Therefore, we deliberately ignore the event types, and we use a single event type, indicating either types of intensity variation. The purpose is to train detectors that are sensitive to certain stimuli sequences. To achieve this, we design and use elementary network structures whose output layer is capable of learning the direction of a given input binary texture in motion.

\section{METHODOLOGY}

We wish to investigate the impact of parameters tuning of a simple feed-forward network on the ability to learn the motion direction of a binary texture pattern. This section relates how the data was generated, gives details of the network structure and default parameters, and describes the experimental protocol.

\subsection{Synthetic event data}

Standard Address Event Representation (AER) encodes positive and negative pixel-wise luminosity variation in the form of $\mathrm{ON}$ - and OFF-events. In our model, we used a simplified representation merging both types into a single type of event. We generated synthetic event data mimicking the displacement of simple texture patterns in four directions (NORTH, SOUTH, WEST, EAST) inside a $5 \times 5$ window. Figure 1 shows an illustration of a square pattern motion in the EAST direction.

Therefore, we have four classes for each pattern. The speed is set to 480 pixels/s as in the experiments described in (Bichler et al., 2012), and one sample input corresponds to a 30-ms duration of stimulation.

We consider several patterns with varying number of input spiking pixels, ranging from 1 to 24 , as shown
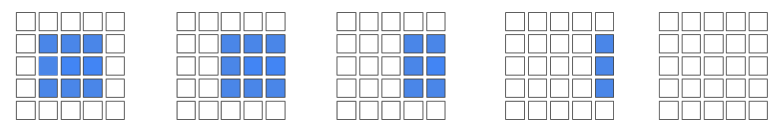

$t=0 \mathrm{~ms}$

$t=3 \mathrm{~ms}$

$t=6 \mathrm{~ms}$

$t=9 \mathrm{~ms}$

$t=12 \mathrm{~ms}$

Figure 1: Illustration of a pattern motion in the EAST direction.

Figure 3.

\subsection{Network details}

Our network is a simple one-layer fully-connected feed-forward SNN, that takes the event data as a $5 \times 5$ continuous input (as illustrated Figure 2), and whose output layer is a vector encoding motion classes.

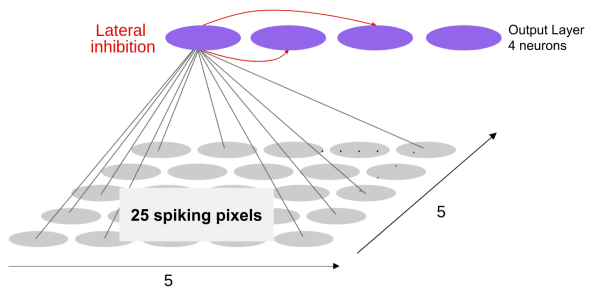

Figure 2: Topological overview of the network.

Among several neuron models, we use the LeakyIntegrate-and-Fire (LIF) model - see (Ponulak and Kasinski, 2011). This model integrates input spikes to its membrane potential $V$. When $V$ exceeds a predefined threshold $V_{\text {thres }}$, then an output spike is triggered and $V$ is reset to 0 . Otherwise, $V$ decays with time (unless other input spikes occur), at a certain rate $\tau_{\text {leak }}$. The model is defined as follows:

$$
\begin{aligned}
\tau_{\text {leak }} \frac{\partial V}{\partial t} & =-V+\sum_{i \in S} V_{i} \delta\left(t-t_{i}\right) \\
V & \leftarrow 0 \text { when } V \geq V_{\text {thres }}
\end{aligned}
$$

$S$ is the set of incoming spikes, $V_{i}$ the voltage of the $i^{t h}$ spike, $t_{i}$ the timestamp of the $i^{t h}$ spike and $\delta$ the Dirac function.

In this model, the synapses modulate the spikes voltage $V_{i}$ that passes through connections according to a synapse weight $w$. The learning rule, common to all the simulations presented in this paper, is a simplified Spike-timing-dependent plasticity (STDP) rule (Bichler et al., 2012).

$$
\begin{aligned}
& \Delta t=t_{\text {post }}-t_{\text {pre }} \\
& \Delta w=\operatorname{STDP}(\Delta t)= \begin{cases}\Delta w_{+} & \text {if } 0 \leq \Delta t \leq T_{L T P} \\
\Delta w_{-} & \text {otherwise }\end{cases}
\end{aligned}
$$


where $t_{\text {pre }}$ (resp. $t_{\text {post }}$ ) represents the presynaptic (resp. postsynaptic) spike timestamp, and $T_{L T P}$ is the width of the potentiation window (LTP stands for Long Term Potentiation). If the postsynaptic spike occurs within $T_{L T P}$ ms after the presynaptic spike, the synapse is potentiated by $\Delta w_{+} \mathrm{mV}$. Otherwise, other values of $\Delta t$ trigger a Long Term Depression (LTD), where the synapse is depressed by $\Delta w_{-} \mathrm{mV}$.

In our experiments, parameters are set individually for each synapse in the following way: a minimum and a maximum bound are set randomly, and the initial value of the synapse is chosen randomly inside the bounds. Moreover, $\Delta w_{-}$and $\Delta w_{+}$are also set randomly for each synapse, with the only constraint that $\Delta w_{-}<\Delta w_{+}$. Moreover, our model includes 3 bio-inspired mechanisms:

- a competition constraint that prevents all output units to learn the same pattern: neurons are equipped with lateral inhibition capability, where an active neuron prevents its neighbours to spike during a given time $T_{\text {inhibit }}$;

- refractory periods to prevent a single output unit to continuously burst without giving other units a chance to emit output spikes;

- synaptic delays to avoid that all incoming spikes occur simultaneously.

We implemented this network using Brian2 SNN simulator (Goodman and Brette, 2009) with parameters similar to (Bichler et al., 2012).

Table 1: Value of the default neuronal parameters

\begin{tabular}{|l|l|l|}
\hline Param. & Value & Description \\
\hline$V_{\text {thres }}$ & $20 \mathrm{mV}$ & Action potential threshold \\
\hline$T_{\text {LTP }}$ & $2 \mathrm{~ms}$ & Long Term Potentiation \\
\hline$T_{\text {refrac }}$ & $10 \mathrm{~ms}$ & Refractory period \\
\hline$T_{\text {inhibit }}$ & $20 \mathrm{~ms}$ & Inhibition time \\
\hline$\tau_{\text {leak }}$ & 5 & Leak rate \\
\hline
\end{tabular}

\subsection{Protocol}

We demonstrate the capabilities for model with several types of input patterns. We show that the model is be able to differentiate between NORTH, SOUTH, WEST, EAST directions for the considered patterns (four distinguishable classes).

During a simulation, we alternate learning phases, corresponding to the period when the network evolves by modifying its weights according to stimuli, and test phases in which the STDP is disabled. It is during the test phases that measurements are made.
Table 2: Mean $(\mu)$ and standard deviation $(\sigma)$ for the synaptic parameters, for all the simulations in this paper. The parameters are randomly chosen for each synapse at the beginning of the simulations, using the normal distribution. Note that the weights are expressed in $\mathrm{mV}$, because it is synapses that deliver tension.

\begin{tabular}{|l|ll|l|}
\hline Param. & $\mu$ & $\sigma$ & Description \\
\hline$w_{\min }$ & $0.02 \mathrm{mV}$ & 0.002 & Minimum weight \\
\hline$w_{\max }$ & $6.00 \mathrm{mV}$ & 2 & Maximal weight \\
\hline$w$ & $50 \%$ & $30 \%$ & $\begin{array}{l}\text { Weight (normalized } \\
\text { with limits) }\end{array}$ \\
\hline$\Delta w_{+}$ & $10 \%$ & $5 \%$ & LTP \\
\hline$\Delta w_{-}$ & $5 \%$ & $2.5 \%$ & LTD \\
\hline
\end{tabular}

We explore the 2 following network parameters : neuron activation threshold and neuron leak. Also, we assess the impact of the number of input spiking pixels $(N)$ by using 24 patterns (see Figure 3 ) where $N$ ranges from 1 to 24 .

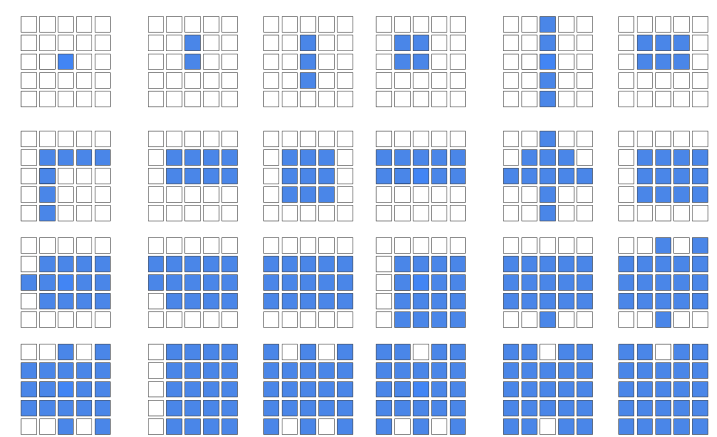

Figure 3: Twenty-four input patterns used in our experiments.

\subsection{Score assessment}

For each simulation, output codes are generated in the following way : an output neuron is assigned 1 if and only if at least one spike is triggered during the test phase. All classes are presented sequentially to the network and we observe the output codes, as illustrated Figure 4. With these output codes, a score can be estimated by comparing the different codes and identifying those that are distinguishable. Indeed, we are interested in distinguishing between input classes. Therefore, the score value is between 0 and 4 .

Note that the output code with only zeros does not represent a class. A score value is obtained during each test phase. Given the random weights initialisation, we run 10 executions per configuration in order to obtain a reliable estimation of the score. There are 40 test phases per run ; for each test phase, the scores 


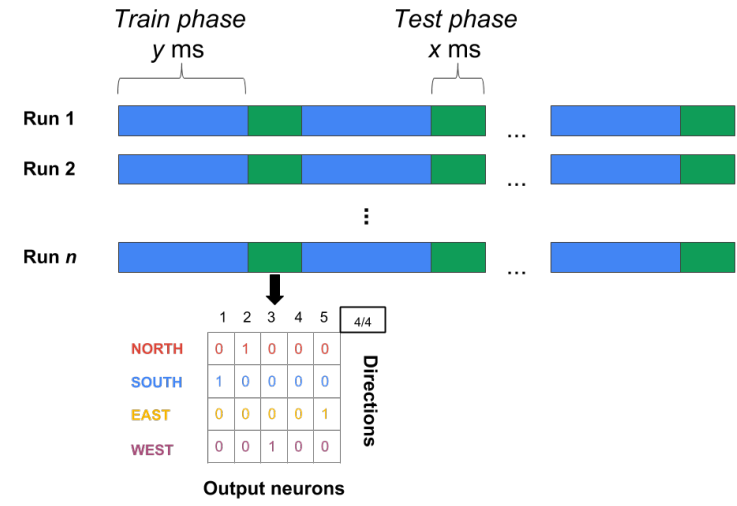

Figure 4: Training phases have a duration of $y \mathrm{~ms}$, they correspond to the presentation of one class. Test phases have a duration of $x \mathrm{~ms}$, they correspond to the presentation of a sequence of all four classes.

of all runs are averaged.

\section{Experimental result}

The aim of the unsupervised training step is to adjust synaptic weights so as to reach a network state where the outputs precisely distinguish between input classes. This means that some output layer neurons would become specialised in detecting a particular input class. A necessary situation of specialisation is when some synaptic weights have converged to values close to 0 or 1 , indicating that the corresponding output neuron has become either insensitive or sensitive to the corresponding input spiking pixel, therefore proving a specialisation of the network toward a particular stimulus.

During simulations, we observe the output activity of the network as well as all the synaptic weight values. In particular, we monitor both output codes for classes during test phases and the synaptic weights evolution (more precisely: the standard deviation of synaptic weights). The aim is to measure the impact of the different parameters of the model during training. During the different experiments, the parameters that do not vary have a value indicated in the Table 1 .

\subsection{Impact of the threshold $V_{\text {thres }}$}

In order to assess the impact of threshold ( $\left.V_{\text {thres }}\right)$, we show the score value when varying $V_{\text {thres }} \in[0,50] \mathrm{mV}$ (Figure 5).

We see that when the threshold has a zero value i.e. all output neurons will emit a spike when receiving a presynaptic spike, regardless of the value of their (strictly positive) synaptic weights. In this

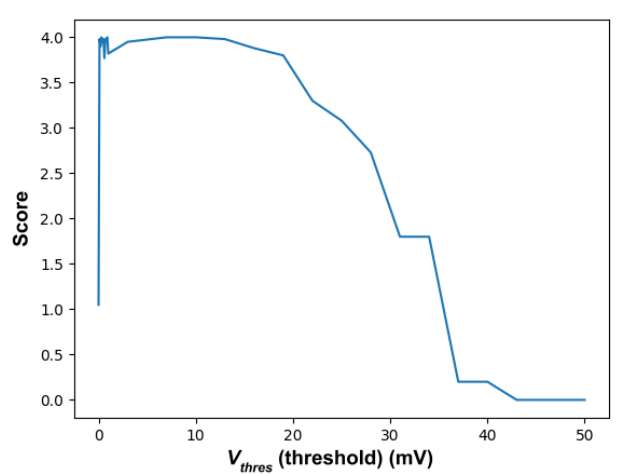

Figure 5: Evolution of the score when varying $V_{\text {thres }}$.

case, the neurons are sensitive to all input stimuli and cannot distinguish between classes, hence the score is 1 (remember that the score denotes the number of classed that can be distinguished). Since the output spikes have no correlation with the incoming spikes, following the STDP learning rule, this configuration results in a succession of LTD decreasing the synaptic connections to such an extent that most values are close to $w_{\text {min }}$. An example of such a situation of low synaptic connection values for a network containing 10 output neurons is displayed Figure 6, where each matrix represents the normalised synaptic weights between one of the 10 output neurons and the 25 input neurons. This representation preserves the spatial arrangement of the input neurons. Light/dark zones indicate weights of sensitive/insensitive connections of output neurons. We can observe that most synaptic weights have become 0 , indicating mainly insensitive output neurons. We also display Figure 7 the evolution curve of the standard deviations of the synaptic weights during the simulation. This curve shows a decreasing standard deviation, which further confirms a wrong parameter setting.

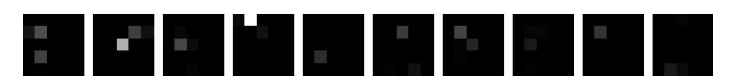

Figure 6: Illustration of synaptic weights for the network when $V_{\text {thres }}=0 \mathrm{mV}$ (non ideal case).

For the following values, in particular, for those between 0.1 and $0.9 \mathrm{mV}$ reach the score of 4 .

For thresholds with high values, the neuron's membrane potential has difficulty reaching this voltage or even more, even in the most favourable configurations (high synaptic weights), which leads to a decrease or even absence of output activity from the network. 


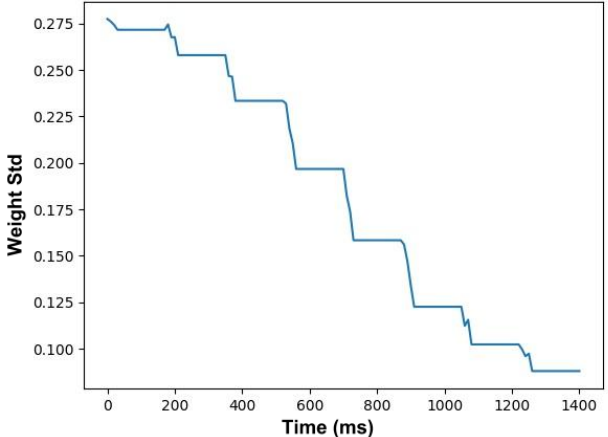

Figure 7: Evolution of the global standard deviation of synaptic weights during a simulation (non ideal case). The plateaus correspond to the different test phases, where the STDP learning rule is disabled.

\subsection{Impact of the leak $\tau_{\text {leak }}$}

The leak can be pictured as a water leak from a pierced bottle. It makes the membrane potential decrease with time, until a rest value is reached. With the water analogy, $\tau_{\text {leak }}$ is a coefficient that would be inversely proportional to the size of the hole in the leaky bottle - see Figure 8.

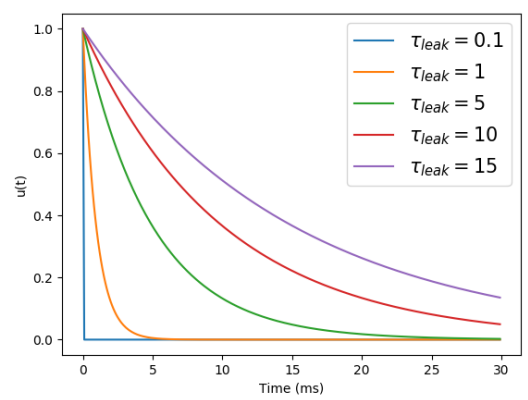

Figure 8: Leak with different values of $\tau_{\text {leak }}$.

In order to assess the impact of neurons' leak, we show in Figure 9 the score values when varying $\tau_{\text {leak }} \in[0,30]$. When $\tau_{\text {leak }}$ is small (resp. large) it means that the leak is fast (resp. slow). When the leak value is low, it causes the obtained score to be also low because the decrease in membrane potential is fast and it prevents the output neurons' membrane to reach the activation threshold. When the leak value is larger, especially when it reaches a certain value $(9$, in our case), the parameter no longer influences the score, that stabilises around the optimal score value of 4. In such case, a LIF neuron with a large leak is an approximation of IF neuron (i.e. a neuron with no leak).

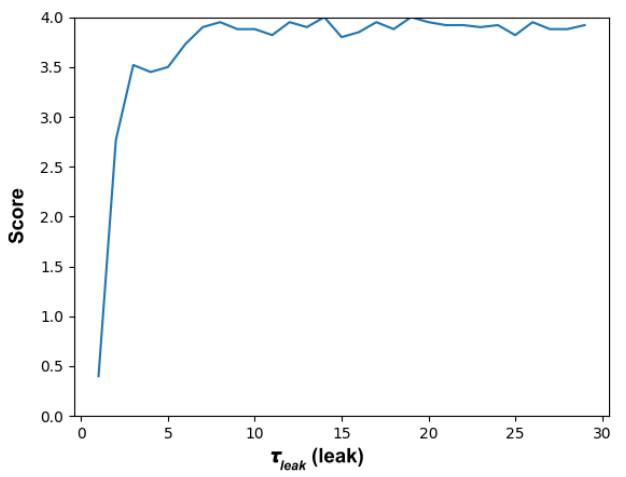

Figure 9: Evolution of the score when varying $\tau_{\text {leak }}$.

\subsection{Impact of the number of input spiking pixels $N$}

In order to assess the impact of the number of input spiking pixels $(N)$, we use the patterns shown Figure 3, where the number of input spiking pixels varies from 1 to 24 . Figure 10 shows the corresponding score values.

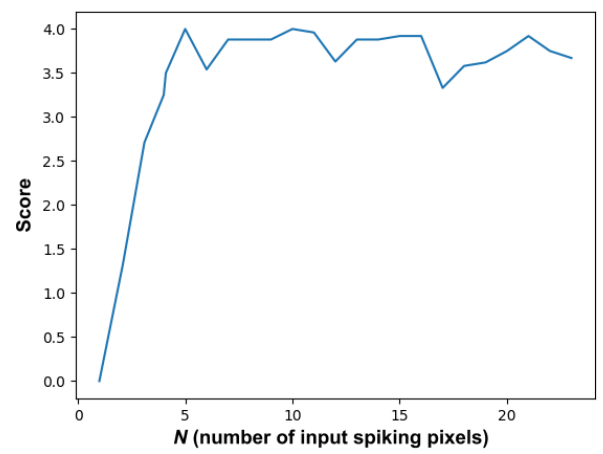

Figure 10: Evolution of the score when varying the number of input spiking pixels $N$.

The number of input spiking pixels $(N)$ has an influence on the score, particularly in situations where the number of pixels is in $[1,3]$, a score is obtained between $[0,3]$. In this case of low $N$ values, the input does not produce enough spikes to activate the output neurons.

\subsection{Joint impact of the threshold $V_{\text {thres }}$ and leak $\tau_{\text {leak }}$}

In order to assess the joint impact of threshold ( $\left.V_{\text {thres }}\right)$ and neuron's leak $\left(\tau_{\text {leak }}\right)$, we show in Figure 11 the score value when varying $V_{\text {thres }} \in[1 \mathrm{mV}, 35 \mathrm{mV}]$ and $\tau_{\text {leak }} \in[0,15]$. 


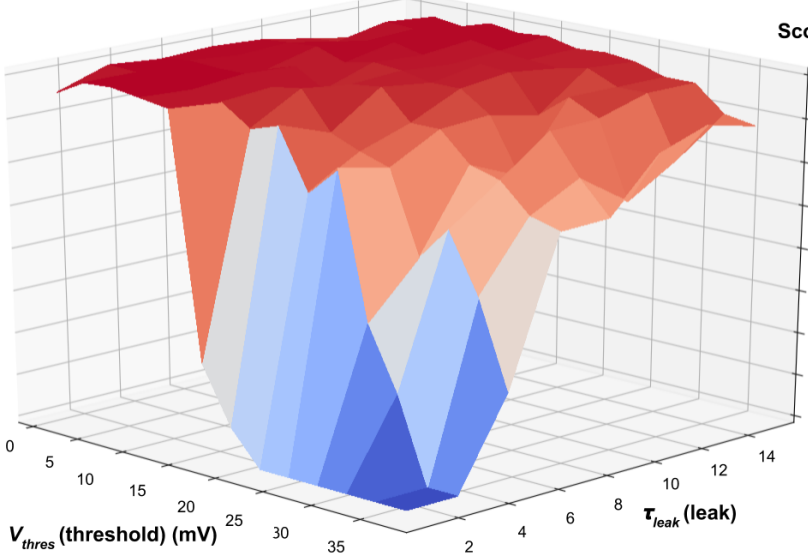

Figure 11: Evolution of the score when varying the threshold $V_{\text {thres }}$ and leak $\tau_{\text {leak }}$.

The same observation can be made as when $\tau_{\text {leak }}$ is low. However, this can be offset by adjusting the threshold to lower values. To support this point, by choosing a threshold $V_{\text {thres }} \in[20 \mathrm{mV}, 35 \mathrm{mV}]$, we observe that by increasing the leak coefficient, the scores improve.

This behaviour comes from the fact that both parameters influence the decision of a neuron to emit a spike or not. The leak controls an aspect of the evolution of the membrane potential and the threshold controls the value that the membrane potential voltage must reach for there to be a spike at the output.

\subsection{Joint impact of the number of input spiking pixels $N$ and threshold $V_{\text {thres }}$}

In order to jointly assess the impact of the number of input spiking pixels $(N)$ and the threshold $\left(V_{\text {thres }}\right)$, we show the score value when varying $N \in[1,24]$ and $V_{\text {thres }} \in[1 \mathrm{mV}, 50 \mathrm{mV}]$ (Figure 12).

When observing the output graph, we see that the parameters configurations yielding low scores are those where $V_{\text {thres }}$ is high and $N$ is small. Indeed, patterns with a number of pixels less than 4 are very sensitive to threshold setting. The best threshold on average is a low threshold at $10 \mathrm{mV}$.

\subsection{Joint impact of the number of input spiking pixels $N$ and leak $\tau_{\text {leak }}$}

In order to jointly assess the impact of the number of input spiking pixels $(N)$ and the leak $\left(\tau_{\text {leak }}\right)$, we show the score value when varying $N \in[1,24]$ and $\tau_{\text {leak }} \in[1,15]$ (Figure 13).

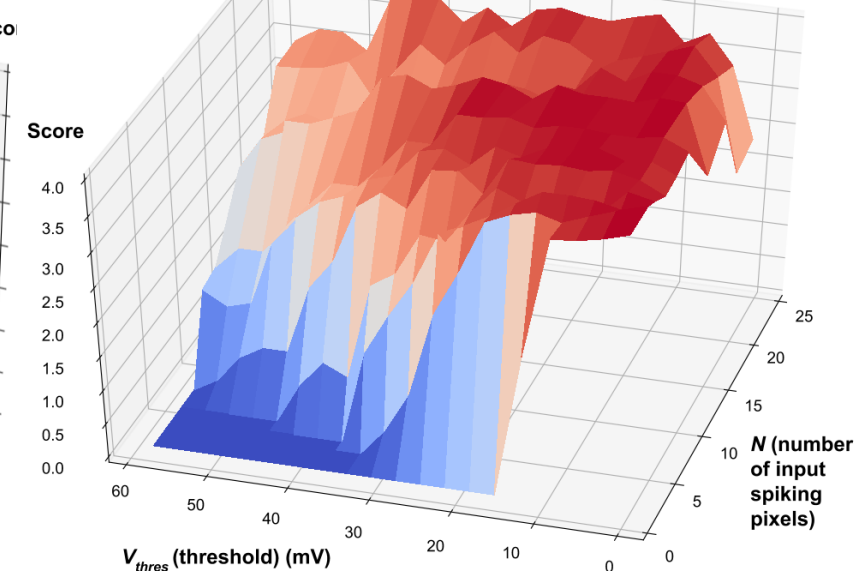

Figure 12: Evolution of the score when varying the number of input spiking pixels $N$ and threshold $V_{\text {thres }}$.

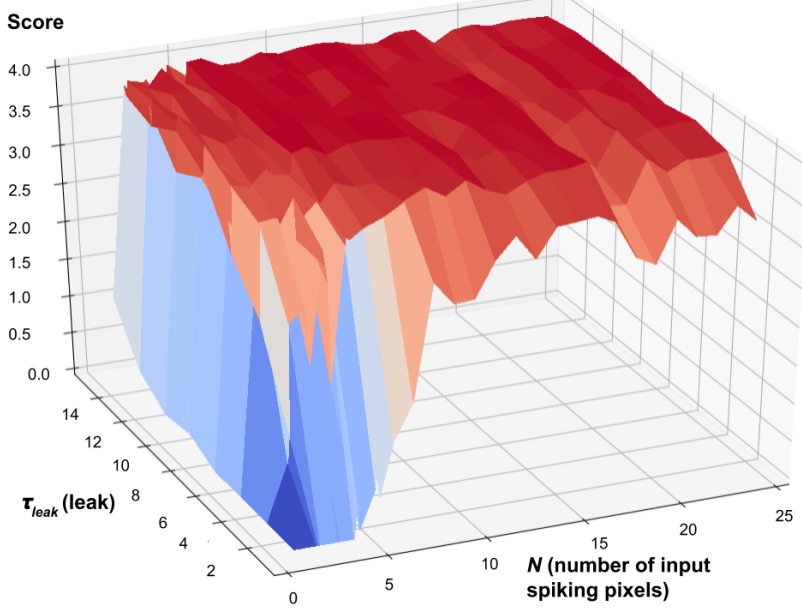

Figure 13: Evolution of the score when varying the number of input spiking pixels $N$ and leak $\tau_{\text {leak }}$.

There is a clear influence of both $N$ and $\tau_{\text {leak }}$ on the output score, even though the impact is less important than the threshold $V_{\text {thres }}$. Most score values are close to 4 , except when $\tau_{\text {leak }}<4$ or $N<2$. We notice that when both $\tau_{\text {leak }}$ and $N$ are small (i.e. fast leak and little input activity), the score becomes null.

\section{CONCLUSION}

In this paper, we have presented a study or metaparameters impact for an approach of unsupervised motion analysis. Our contributions include the design and training of a Spiking Neural Network to classify event video data, with a parameter exploration regarding the threshold $V_{\text {thres }}$, the leak leak $\tau_{\text {leak }}$, and the 
number of input spiking pixels $N$. The aims is to estimate the impact of the parameters $N, V_{\text {thres }}$ and $\tau_{\text {leak }}$ on the model for the motion classification task.

In future work, we wish to explore two orthogonal directions:

- target invariance regarding motion speed, possibly by further exploiting synaptic delays so that several speeds will trigger the same network output,

- target speed sensitivity, with a dedicated architecture whose output units are specialised for distinct speed classes.

We will refine the method of evaluating the efficiency of the network, using a classifier on the output spikes. Also, we will evaluate our model on real-world event data.

In a longer term, we wish to use such elementary networks as local motion detectors, to be laid out in layers or other topologies to enable unsupervised analysis of more complex motion patterns.

\section{ACKNOWLEDGEMENTS}

This work has been partly funded by IRCICA (Univ. Lille, CNRS, USR 3380 IRCICA, Lille, France).

\section{REFERENCES}

Amir, A., Taba, B., Berg, D. J., Melano, T., McKinstry, J. L., Di Nolfo, C., Nayak, T. K., Andreopoulos, A., Garreau, G., Mendoza, M., et al. (2017). A low power, fully event-based gesture recognition system. In $C V P R$, pages 7388-7397.

Bichler, O., Querlioz, D., Thorpe, S. J., Bourgoin, J.-P., and Gamrat, C. (2012). Extraction of temporally correlated features from dynamic vision sensors with spike-timing-dependent plasticity. Neural Networks, 32:339-348.

Cao, Y., Chen, Y., and Khosla, D. (2015). Spiking deep convolutional neural networks for energy-efficient object recognition. International Journal of Computer Vision, 113(1):54-66.

Goodman, D. F. and Brette, R. (2009). The brian simulator. Frontiers in neuroscience, 3:26.

Hopkins, M., Pineda-García, G., Bogdan, P. A., and Furber, S. B. (2018). Spiking neural networks for computer vision. Interface Focus, 8(4):20180007.

Ilg, E., Mayer, N., Saikia, T., Keuper, M., Dosovitskiy, A., and Brox, T. (2017). Flownet 2.0: Evolution of optical flow estimation with deep networks. In CVPR 2017, pages 1647-1655. IEEE.

Kreiser, R., Moraitis, T., Sandamirskaya, Y., and Indiveri, G. (2017). On-chip unsupervised learning in winnertake-all networks of spiking neurons. In Biomedi- cal Circuits and Systems Conference (BioCAS), 2017 IEEE, pages 1-4. IEEE.

Lee, J. H., Delbruck, T., and Pfeiffer, M. (2016). Training deep spiking neural networks using backpropagation. Frontiers in neuroscience, 10:508.

Li, H., Liu, H., Ji, X., Li, G., and Shi, L. (2017). Cifar10dvs: an event-stream dataset for object classification. Frontiers in neuroscience, 11:309.

Lichtsteiner, P., Posch, C., and Delbruck, T. (2008). A $128 \times 128120 \mathrm{db} 15 \mu$ s latency asynchronous temporal contrast vision sensor. IEEE journal of solid-state circuits, 43(2):566-576.

Maass, W. (1997). Networks of spiking neurons: the third generation of neural network models. Neural networks, 10(9):1659-1671.

Masquelier, T. and Thorpe, S. J. (2007). Unsupervised learning of visual features through spike timing dependent plasticity. PLoS computational biology, 3(2): 31 .

Merolla, P. A., Arthur, J. V., Alvarez-Icaza, R., Cassidy, A. S., Sawada, J., Akopyan, F., Jackson, B. L., Imam, N., Guo, C., Nakamura, Y., et al. (2014). A million spiking-neuron integrated circuit with a scalable communication network and interface. Science, 345(6197):668-673.

Orchard, G., Benosman, R., Etienne-Cummings, R., and Thakor, N. V. (2013). A spiking neural network architecture for visual motion estimation. In Biomedical Circuits and Systems Conference (BioCAS), 2013 IEEE, pages 298-301. IEEE.

Orchard, G., Jayawant, A., Cohen, G. K., and Thakor, N. (2015). Converting static image datasets to spiking neuromorphic datasets using saccades. Frontiers in neuroscience, 9:437.

Oudjail, V. and Martinet, J. (2019). Bio-inspired eventbased motion analysis with spiking neural networks. In VISAPP 2019, pages 389-394.

Paugam-Moisy, H. and Bohte, S. (2012). Computing with spiking neuron networks. In Handbook of natural computing, pages 335-376. Springer.

Ponulak, F. and Kasinski, A. (2011). Introduction to spiking neural networks: Information processing, learning and applications. Acta neurobiologiae experimentalis, 71(4):409-433.

Serrano-Gotarredona, T. and Linares-Barranco, B. (2015). Poker-dvs and mnist-dvs. their history, how they were made, and other details. Frontiers in neuroscience, 9:481.

Sourikopoulos, I., Hedayat, S., Loyez, C., Danneville, F., Hoel, V., Mercier, E., and Cappy, A. (2017). A 4fj/spike artificial neuron in $65 \mathrm{~nm}$ cmos technology. Frontiers in neuroscience, 11:123.

Tavanaei, A. and Maida, A. S. (2017). Multi-layer unsupervised learning in a spiking convolutional neural network. In IJCNN 2017, pages 2023-2030. IEEE.

Zhao, B., Ding, R., Chen, S., Linares-Barranco, B., and Tang, H. (2015). Feedforward categorization on aer motion events using cortex-like features in a spiking neural network. IEEE Trans. Neural Netw. Learning Syst., 26(9):1963-1978. 